They question whether we were able to empty the balloon between inflations. Because we measured the volume inserted into the balloon on each occasion and also the volume recovered we were able to ensure that accumulation of fluid in the balloon did not occur. They criticise the use of a fluid filled catheter to detect intraballoon pressure (standard practice by most other workers). We agree that different absolute values could be obtained using different catheters but find it difficult to see how the varying inter and intraindividual results we obtained could be the result of using different catheters.

They also question whether our normal subject group, all of whom were symptom free and without past or current history of bowe symptoms, could have inadvertently included subjects with irritable bowel syndrome. If it is possible for an adult who feels healthy and who is not aware of any symptoms referable to the gastrointestinal tract to be suffering from irritable bowel syndrome then we have to admit guilt, but then anybody studying norma human physiology of the gut will commit the same mistake.

Varma and Smith also make further points concerning irritable bowel syndrome, suggesting that there may be subgroups who could perform differently. We would not disagree with this possibility and indeed two of our patients with predominant diarrhoea (cases 4 and 24) had maximal tolerable volumes to distension which were at the bottom of the patient range. The point that we made in our paper, however, is that neither of these two patients fell outside the $95 \%$ confidence limits for our normal range and while there may indeed be a difference between irritable bowel syndrome patients who are at the extremes of symptoms, neither of these two extremes can be regarded as being 'abnormal.'

They question the past history of the patients and their age: we also considered this and mentioned it in our discussion. It seems likely that failure to control for differences between age and sex of the two groups would have increased the differences between the groups rather than reduced them.

They comment that rectal volumes tolerated by subjects would vary with the confidence and experience of the subjects. We wholeheartedly agree with this statement and indeed this was one of the aims of our study. Our results showed that variability progressively reduced in an individual as the study continued. This is discussed in detail in our paper.

They go on to mention that individual variability would not be so great if other sensory or motor indices of rectal distension were used. The major difficulty identified and discussed in our paper is that the proctometrogram as traditionally employed relies on sensory end points, which are subject to individual interpretation rather than independent measurement.

They comment that the tracings which we published of the pressure-volume relations seem to be consistent in each individual and indeed we would agree with this. Our point again, however, is that it is the sensory end points that are variable, even though the pressure-volume curve is consistent. The major problem in analysis of pressure-volume curves, however, is to adequately define in mathematical terms the pressure-volume curve itself. It is of doubtful use to talk about compliance (as do many workers in the field), since as is evident from all published data, the slope of the pressure-volume curve varies during inflation so that there are a number of compliancies rather than a single value.
Sun et al also criticise our selection of patients and suggest that our sample was biased towards constipation. Thirteen of 26 patients were constipated, two were predominately diarrhoea sufferers, five had both diarrhoea and constipation, the rest were unaffected by major alterations in stool consistency. These patients therefore do not seem too different from those reported in other recent studies of irritable bowel syndrome such as that of Prior et al (Sun et al, reference 1) in which 27 of 55 irritable bowel patients were constipated. We would not argue with their suggestion that patients with diarrhoea predominant symptoms may differ from those with constipation predominant symptoms and also are aware that those individuals with diarrhoea in our study had the lowest tolerated volumes of the groups. We point out again, however, that none of these individuals fell outside the range of normality as defined by our volunteer data, so that while proctometrography may differentiate between irritable bowel syndrome patients with diarrhoea and constipation, it is difficult to see how the technique could be used to distinguish the irritable bowel syndrome from normal.

From the interest which our article has stimulated it is evident that more data are required to understand the basic factors influencing pressure-volume relations in the rectum. We look forward to seeing more data from both correspondents to help clarify these difficulties.

D G THOMPSON
Department of Medicine,
Section of Gastroenterology,
Hope Hospital,
Eccles Old Road,
Manchester M6 8HD

$\star$ This is an abbreviated version. The original version addressed all the points made. $E d$.

\section{Why do patients with ulcerative colitis relapse?}

SIR, - Dr J W Paulley takes issue with our criticism that previous studies of psychological factors and ulcerative colitis relapse are uncontrolled (Gut 1990; 31: 1419). In support of his argument he cites three studies in which radiotherapy patients, healthy siblings, and healthy volunteers are used as 'control' groups against which ulcerative colitis patients are compared. The data as presented by Dr Paulley are, however, misleading. In the first study radiotherapy patients were only used as controls for the purpose of personality comparisons. The data concerning colitis relapse are uncontrolled and anecdotal. The second study ${ }^{2}$ is a comparison of personality characteristics between 23 patients with inflammatory bowel disease (12 with ulcerative colitis) and their healthy siblings and makes no attempt to study colitis relapse. This study is considerably flawed since the decision to use sibling controls was based on previous data suggesting that siblings had different personalities. Unfortunately, we have been unable to obtain a copy of the 1957 Czech study ${ }^{3}$ as there is no United Kingdom source (British Library, personal communication). We suspect, from his limited description, that this too is a personality assessment.

Unfortunately, personality studies are of limited value in the study of ulcerative colitis as it is difficult to distinguish premorbid characteristics from illness related changes. It is clearly inappropriate to use controls who do not suffer from episodic bloody diarrhoea and who are not at risk of incontinence, surgery, and cancer. We recognised that matched disease controls are not easy to find but the above studies are inappropriately controlled.

The relapsing nature of colitis offers an ideal opportunity to undertake controlled studies of the psychological factors associated with relapse. Patients in relapse may be compared with those in remission and in cohort studies patients may act as their own controls. Using such a design we found that $20 \%$ of patients reported stressful life events in the three months before relapse. In the absence of controlled data this suggests an association, but patients in remission reported a similar number of events. ${ }^{4}$

Finally, Dr Paulley believes that in forming our conclusion that psychological factors are unimportant in colitis relapse we have 'listened to commonly recited, but uncorroborated views of others, rather than checked the original sources.' We suggest that such criticisms are commonly voiced because others have also checked the original studies and found them lacking.

$$
\begin{array}{r}
\text { S A RILEY } \\
\text { Hope Hospital, Salford } \\
\text { V MANI } \\
\text { Leigh Infirmary } \\
\text { M J GOODMAN } \\
\text { Bury General Hospital, Manchester }
\end{array}
$$

1 Paulley JW. Ulcerative colitis: a study of 173 cases. Gastroenterology 1959; 16: 566-76.

2 McMahon AW, Schmitt P, Paterson JF. Personality differences between inflammatory bowel ality differences between inflammatory bowel Psychosom Med 1973; 35: 91-103.

3 Roubicek J, Martonova F. Psychopathology of ulcerative colitis. Cesk Psychiatr 1957; 53: 220 30.

4 Riley SA, Mani V, Goodman MJ, Lucas S. Why do patients with ulcerative colitis relapse? Gut 1990; 31: 179-83.

\section{Sedation for upper gastrointestinal endoscopy}

SIR,-The paper by Daneshmend et al (Gut $1991 ; 32: 12-5$ ), outlining the results of a postal questionnaire from 665 endoscopists, provides some alarming statistics - 52 deaths, and a further 119 respiratory arrests and 37 cardiac arrests in a two year period.

I suggest that most of these incidents could have been avoided if the following precautions had been observed: (a) all patients were monitored with pulse oximetry; (b) all patients received supplemental oxygen; (c) a trained medical observer devoted total attention to the sedation and cardiorespiratory well being of the patient; (d) oxygen was continued in the postendoscopy period when indicated.

In the experience of many endoscopy units a combination of midazolam and fentanyl given in doses appropriate to the age and condition of the patient provides satisfactory sedation that is safe provided that the above precautions are observed.

It is a pity that some endoscopy units seem to be isolated from departments of anaesthesia, who may be able to help with advice and trained personnel to make the procedure safer.

$$
\begin{array}{r}
\text { R CLARK } \\
\text { J GOY } \\
\text { Dandenong Gastrointestinal Clinic, } \\
\text { Melbourne, Australia }
\end{array}
$$

SIR, - I was interested that in the results of the reported study of postal questionnaires inquiring about the sedation practice of endoscopy clinicians only $2 \%$ of respondents stated they did not use sedation. It has been my practice since 1976 to offer no sedation to patients but 